\title{
Molecular Epidemiology of Cryptosporidiosis in Children in Malawi
}

\author{
MICHAEL M. PENG, ${ }^{\text {a,b }}$ STEVE R. MESHNICK, ${ }^{\text {h.c }}$ NIGEL A. CUNLIFFE, ${ }^{d, c}$ BENSON D.M. THINDWA, \\ C. ANTHONY HART, ${ }^{\circ}$ ROBIN L. BROADHEAD ${ }^{f}$ and LIHUA XIAO ${ }^{a}$ \\ ${ }^{a}$ Centers for Disease Control and Prevention, Atlanta, GA, ${ }^{\mathrm{b}}$ University of Michigan, Ann Arbor, MI, and \\ ${ }^{\mathrm{c}}$ University of North Carolina, Chapel Hill, NC, and ${ }^{\mathrm{d}}$ Wellcome Trust Research Laboratories, Blantyre, Malawi, and \\ 'University of Liverpool, Liverpool, $U K$, and 'University of Malawi, Blantyre, Malawi
}

\begin{abstract}
Few studies have examined the molecular epidemiology of cryptosporidiosis in developing countries. In this study, DNA of 69 microscopy-positive human fecal samples collected from Malawi were examined by multilocus genetic analyses. From 43, 27 and 28 of the samples, the SSU rRNA, $70 \mathrm{kDa}$ heat shock protein (HSP70) and $60 \mathrm{kDa}$ glycoprotein (GP60) genes, respectively, were successfully PCR-amplified. Restriction analysis of the SSU PCR products showed that 41 of the 43 PCR-positive samples had C. hominis and 2 had C. parvum. Sequence analysis of the HSP70 and GP60 gene confirmed the species identification by SSU rRNA PCR-RFLP analysis, but also revealed high intraspecific variations. Altogether, six HSP70 subtypes and six GP60 subtypes (belonging to four subtype alleles) of $C$. hominis were found. Linkage disequilibrum analysis of the two genetic loci showed possible intraspecific recombination. Thus, cryptosporidiosis in the study area was largely caused by anthroponotic transmission. The high intraspecific variation and existence of genetic recombination were probably results of high transmission of cryptosporidiosis in this area.
\end{abstract}

\section{INTRODUCTION}

Molecular epidemiologic studies of cryptosporidiosis are still in their infancy, but significant progress has been made toward better understanding of the transmission of cryptosporidiosis in humans and the public health significance of Cryptosporidium parasites from farm animals, companion animals, and birds. Thus far, five Cryptosporidium spp., C. hominis, C. parvum, C. meleagridis, C. felis, and $C$. canis, have been shown to be responsible for most human infections, with the former two being most common [15]. Because these five parasites have different host specificities, the identification of Cryptosporidium to species level is needed to characterize the transmission dynamics and to infer infection sources. Recently, several subtyping tools have been developed, which allows investigators to address these issues in greater depth [1, 5, 8, 9, 11-13].

Between 1997 and 1999, 69 Cryptosporidium positive samples were collected from Malawian children, and analyzed using both genotyping and subtyping tools. Genotyping and subtyping results were analyzed with clinical and demographic data. Even though there was no association between genotype or subtypes and any of the clinical and demographic profiles analyzed, the predominance of $C$. hominis in the study population suggested that anthroponotic transmission was probably the major transmission route in Malawi.

\section{MATERIALS AND METHODS}

Origin and preparation of parasites. Sixty-nine stool samples were collected from children who visited the Queen Elizabeth Central Hospital (QECH)in Blantyre between August 1997 and March 1999. These children were diagnosed as positive for Cryptosporidium by microscopic examinations of acid-fast stained fecal smears. Forty-five of the children were hospitalized patients, whereas the rest were outpatients. Thirteen of the patients were seropositive for HIV-1 and 45 were HIV-seronegative; the HIV infection statuses for the remaining 11 patients were not known. Some of the patients were enrolled in a previously published study [4]. Samples were stored at $-20^{\circ} \mathrm{C}$ before DNA extraction.

Molecular analysis. DNA was extracted from all samples by an established method [14]. All samples were initially genotyped by a small subunit (SSU) rRNA-based PCR-RFLP technique [14]. Samples that were positive for Cryptosporidium spp. by the SSU rRNA PCR were further subtyped by DNA sequencing of the PCR products of the 70-kilodalton heat shock protein (HSP70; $\sim 1,800 \mathrm{bp}$ ) and the 60-kilodalton glycoprotein (GP60; $\sim 900 \mathrm{bp}$ ) [1,13]. For phylogenetic analysis, a neighbor-joining tree was constructed using the program TreeCon (http://www.psb.rug.ac.be/bioinformatics/psb/ Userman/treeconw.html) based on genetic distances calculated with

Corresponding author: L. Xiao. Telephone: 770-488-4840; Fax: 770-4884454; Email: 1xiao@cdc.gov the Kimura 2-parater model. The HSP70 tree was rooted using a sequence from C. parvum (A53), whereas the GP60 tree was rooted using a sequence from $C$. meleagridis (GenBank AF401499). Nucleotide sequences reported in this study were deposited in the GenBank database under accession numbers AY380458-AY380459 and AY382668-AY382675.

\section{RESULTS AND DISCUSSION}

Distribution of cryptosporidiosis cases by age and season. All samples used in this study were collected from children between the ages of 1 to 30 months in the city of Blantyre, Malawi between 1997 and 1999. Most of the cases occurred in children younger than 1.5 years, with a median age of 10 months (Fig. 1A). The distribution of the cases peaked between October and March of each of the two years (Fig. 1B). These months approximately corresponded to the rainy season in this region. Conversely, during the dry season months, the number of cases dropped. During January of each year in the collection period, the number of cases decreased. This could be the result of the mandatory civil holiday from December 15 to January 5 .

The early occurrence of Cryptosporidium infection in Malawian children suggests that there is intensive Cryptosporidium transmission in Malawi. The study children had a peak occurrence of cryptosporidiosis at 10 months of age, which is similar to the results of study in Mexico [7] but much earlier than those reported in other areas [2,3,10]. It remains to be determined whether the differences were due to small sample size in this study. The seasonal transmission of Cryptosporidium infection and its association with rainfall have been previously reported $[3,6,7]$.

Cryptosporidium genotypes. Out of the 69 isolates analyzed, 43 samples were amplified by PCR using the SSU rRNA primers. The samples were frozen and thawed several times during their transfer. This could have resulted in some DNA degradation, and made it impossible to remove the PCR inhibitors from the samples by washing before DNA extraction. Thus, only 43 of the 69 samples could be amplified by the SSU rRNA PCR. Surprisingly, repeated PCR analyses of multiple extractions of the same samples and the use of bovine serum albumin (a PCR facilitator in samples with inhibitors) failed to produce more SSU rRNA PCR amplification in PCR negative samples.

RFLP analyses of SSU rRNA PCR products with restriction enzymes $S s p$ I and $V s p$ I showed that the $C$. hominis was the predominant parasites; 41 of 43 the patients were infected with C. hominis and 2 of 43 the patients were infected with C. parvum. All of the six PCR-positive samples from HIV-1 positive children had C. hominis. Several other common species such as $C$. meleagridis, C. felis and $C$. canis were not detected. Thus, results of the study indicated that anthroponotic transmission played a major role in the epidemiology of cryptosporidiosis in children in Malawi. This is in 

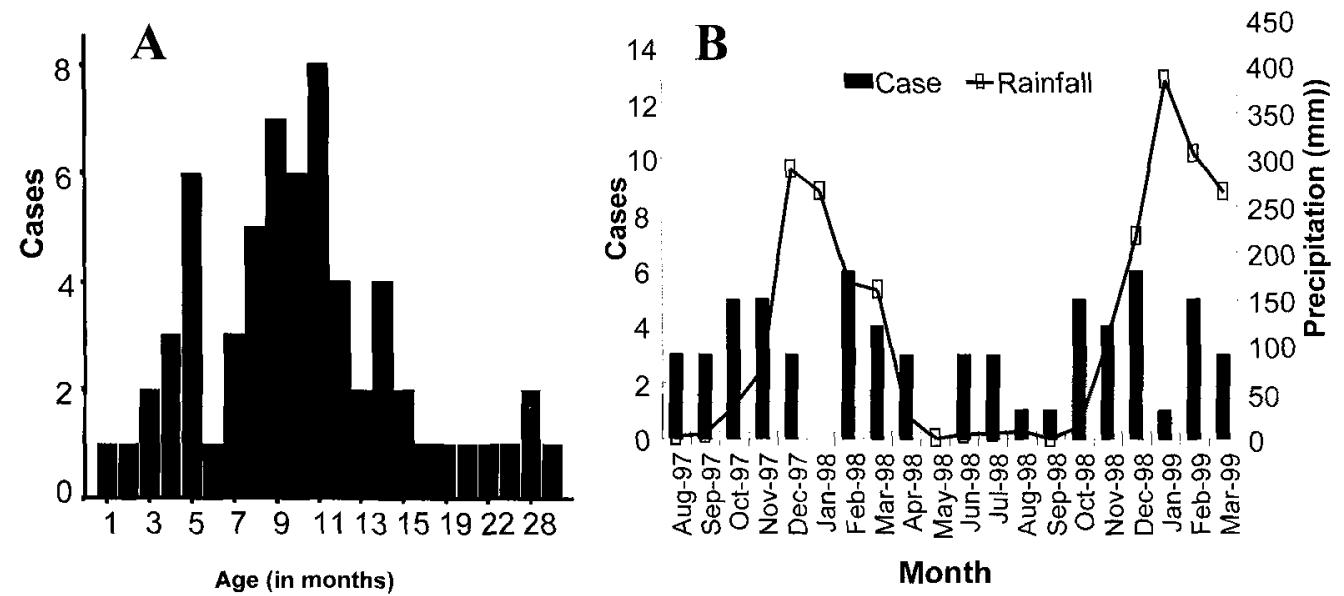

Fig. 1. Age (a) and seasonal (b) distribution of Cryptosporidium cases in Malawian children. Precipitation data were from the Malawi Meteorology Center in Chileka Airport, Blantyre.

agreement with observations made in the United States, Australia, Kenya, Thailand and South Africa where anthroponotic parasites are also responsible for the majority of cases of cryptosporidiosis. In contrast, in European countries (United Kingdom, Switzerland, France, and Portugal), C. parvum are responsible for more infections than C. hominis [15].

HSP70 Subtypes. Twenty-six of the 41 C. hominis-positive samples and 1 of the $2 C$. parvum-positive samples were amplified by HSP70 PCR. DNA sequencing of the PCR products showed the presence of six $C$. hominis subtypes (subtypes A, C, D, E, G, and H), which differed from each other at seven polymorphic sites in the second half of the gene. There was no difference in the number of the 12-bp repeats among the six subtypes, as previously shown among some $C$. hominis subtypes [13]. Four of the subtypes were previously reported before [13], but $\mathrm{G}$ and $\mathrm{H}$ were new HSP70 subtypes. Two subtypes previously detected in North and South America [13], B and $\mathrm{F}$, were not found in Malawi. Most of the isolates found in this study belonged to subtypes $\mathrm{C}$ and $\mathrm{E}$, which each had nine samples (Fig. 2A). Other subtypes had only 1-3 samples. There was no age or season associated predominance of HSP70 subtypes. There was also no

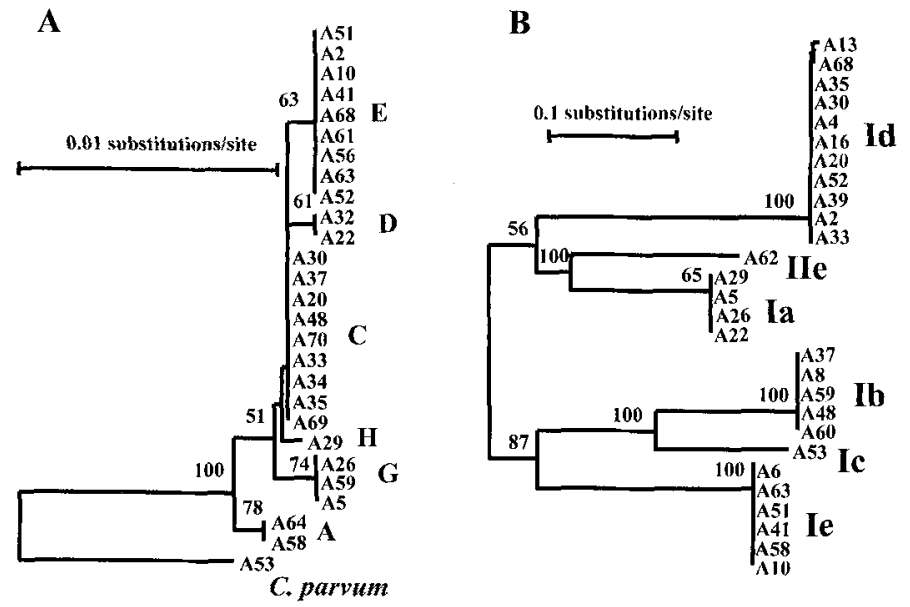

Fig. 2. The presence of multiple C. hominis subtypes in Malawian children as revealed by neighbor-joining analyses of the sequences of HSP70 (a) and GP60 (b). HSP70 subtypes are labeled as A to H. Ia, Ib, Id and Ie are $C$. hominis GP60 allele families; Ic and IIe are $C$. parvum GP60 allele families. association between HSP70 subtypes and clinical presentations (HIV infection status, hospitalization, intravenous rehydration, etc.).

Few data are available on the distribution of $C$. hominis HSP70 subtypes. However, the single nucleotide polymorphisms in the HSP70 gene identified in this study are similar to those we identified in a previous study with samples from the United States and some other areas [13], indicating that these HSP70 subtypes are probably also widely distributed. Indeed, the two most common HSP70 subtypes in Malawi, C and E, were previously found in the United States, Guatemala, and Australia [13].

GP60 Subtypes. Twenty-six of the $41 C$. hominis-positive samples and both of the C. parvum-positive samples were amplified by GP60 PCR. DNA sequencing of the PCR products showed the presence of six subtype allele families: allele families of $\mathrm{Ia}, \mathrm{Ib}, \mathrm{Id}$ and $\mathrm{Ie}$ of $C$. hominis and Ic and IIe of C. parvum (Fig. 2B). With the exception of allele family Id, which had three subtypes, all other allele families had only one subtype sequence. The $C$. hominis allele families ( $\mathrm{Ia}, \mathrm{Ib}, \mathrm{Id}$, and Ie) all had multiple isolates $(4,5,11$, and 6 isolates, respectively). There was no age or season associated predominance of GP60 subtypes. There was also no association between GP60 subtypes and clinical presentations. The $C$. hominis GP60 subtype families identified in this study are common in humans and have been reported in many other parts of the world, such as the United States, United Kingdom, Peru, Portugal and South Africa [1,5,8,11-13].

PCR analyses of the HSP70 and GP60 genes are known to have much lower sensitivities than the SSU rRNA PCR due to the single copy nature of the HSP70 and GP60 genes, larger amplicons, and possible primer mismatches $[1,13]$. Thus, only 27 or 28 SSU rRNApositive samples were amplified in subtyping analysis.

Linkage between HSP70 and GP60 subtypes. Concordant genotyping results were obtained at the SSU rRNA, HSP70 and GP60 genes for all isolates that could be amplified by locus-specific PCR. However, linkage disequilibrium analysis revealed the existence of discordance in subtyping results of $C$. hominis between HSP70 and GP60 loci. Eighteen C. hominis samples were subtyped at both HSP70 and GP60 loci. HSP60 subtype E isolates had two GP60 subtypes (Id and Ie), but one of the GP60 subtype (Id) was also found in isolates with HSP70 subtype C. Likewise, GP60 subtype Ia was found in three HSP70 subtypes (D, G, and H) (Table 1).

Unlike in a previous study where a strong linkage disequilibrium between HSP70 and GP60 subtypes was observed [13], a high discordance in subtyping results between HSP70 and GP60 loci was observed in this study. This difference is probably due to the nature of samples used in these two studies. In the previous study, samples were 
Table 1. Discordance between subtyping results of $C$. hominis isolates between the HSP70 and GP60 loci.

\begin{tabular}{lcc}
\hline \hline Sample & HSP70 subtype & GP60 subtype \\
\hline A58 & A & Ie \\
A30 & C & Id \\
A33 & C & Id \\
A35 & C & Id \\
A37 & C & Ib \\
A48 & C & lb \\
A22 & D & Ia \\
A2 & E & Id \\
A10 & E & Ie \\
A41 & E & I \\
A51 & E & Ie \\
A52 & E & Id \\
A63 & E & Ie \\
A68 & E & Id \\
A5 & G & Ia \\
A26 & G & Ia \\
A59 & G & Ib \\
A29 & H & la \\
\hline
\end{tabular}

largely from outbreaks in the United States, which probably have a more clonal population structure. The lack of linkage in $C$. hominis between HSP70 and GP60 subtyping in Malawi is probably an indication for genetic recombination. A recent study in the United Kingdom has also shown a lack of linkage disequilibrium in both $C$. parvum and $C$. hominis among multiple genetic loci [9]. Thus, intraspecific recombination in Cryptosporidium is probably more common than previously believed. The high genetic heterogeneity and genetic recombination in $C$. hominis are probably indictors of high transmission of cryptosporidiosis in the study area.

Implications. Despite the limited number of samples and failures in amplifying some samples by PCR, results of this study showed the presence of extensive genetic heterogeneity in Cryptosporidium spp. in Malawi, especially at the subtype level. This plus the existence of genetic recombination in C. hominis and the early occurrence of cryptosporidiosis in children suggest there is intensive transmission of Cryptosporidium infections in Malawi. Nevertheless, the predominance of $C$. hominis in study children indicates that anthroponotic route plays a major role in Cryptosporidium transmission in Blantyre, Malawi.

\section{ACKNOWLEDGMENTS}

This work received financial support from the Wellcome Trust of Great Britain (grant number 049485/Z/96) and forms part of the Malawi-Liverpool-Wellcome Trust Programme of Research in Clinical Tropical Medicine.

\section{LITERATURE CITED}

1. Alves, M., Xiao, L., Sulaiman, I., Lal, A.A., Matos, O. \& Antunes, F. 2003. Subgenotype analysis of Cryptosporidium isolates from humans, cattle, and zoo ruminants in Portugal. J. Clin. Microbiol., 41:2744-2747.
2. Bern, C., Hernandez, B., Lopez, M.B., Arrowood, M.J., De Merida, A.M. \& Klein, R.E. 2000. The contrasting epidemiology of Cyclospora and Cryptosporidium among outpatients in Guatemala. Am. J. Trop. Med. Hyg., 63:231-235.

3. Bhattacharya, M.K., Teka, T., Faruque, A.S. \& Fuchs, G.J. 1997. Cryptosporidium infection in children in urban Bangladesh. J. Trop. Pediatrics, 43:282-286.

4. Cunliffe, N.A., Gondwe, J.S., Kirkwood, C.D., Graham, S.M., Nhlane, N.M., Thindwa, B.D.M., Dove, W., Broadhead, R.L., Molyneux, M.E. \& Hart, C.A. 2001. Effect of concomitant HIV infection on presentation and outcome of rotavirus gastroenteritis in Malawian children. Lancet, 358:550-555.

5. Glaberman, S., Moore, J.E., Lowery, C.J., Chalmers, R.M., Sulaiman, I., Elwin, K., Rooney, P.J., Millar, B.C., Dooley, J.S., Lal, A.A. \& Xiao, L. 2002. Three drinking-water-associated cryptosporidiosis outbreaks, Northem Ireland. Emerg. Infect. Dis., 8:631-633.

6. Iqbal, J., Hira, P.R., Al-Ali, F. \& Philip, R. 2001. Cryptosporidiosis in Kuwaiti children: seasonality and endemicity. Clin. Microbiol. Infect., 7:261-266.

7. Javier Enriquez, F., Avila, C.R., Ignacio Santos, J., Tanaka-Kido, J., Vallejo, O. \& Sterling, C.R. 1997. Cryptosporidium infections in Mexican children: clinical, nutritional, enteropathogenic, and diagnostic evaluations. Am. J. Tmp. Med. Hyg, 56:254-257.

8. Leav, B.A., Mackay, M.R., Anyanwu, A., O'Connor, R.M., Cevallos, A.M., Kindra, G., Rollins, N.C., Bennish, M.L., Nelson, R.G. \& Ward, H.D. 2002. Analysis of sequence diversity at the highly polymorphic Cpgp40/15 locus among Cryptosporidium isolates from human immunodeficiency virus-infected children in South Africa. Infect, Immun., 70:3881-3890.

9. Mallon, M., MacLeod, A., Wastling, J., Smith, H., Reilly, B. \& Tait, A. 2003. Population structures and the role of genetic exchange in the zoonotic pathogen Cryptosporidium parvum. J. Mol. Evol, 56:407-417.

10. Newman, R.D., Sears, C.L., Moore, S. R., Nataro, J.P., Wuhib, T., Agnew, D.A., Guerrant, R.L. \& Lima, A.A.M. 1999. Longitudinal study of Cryptosporidium infection in children in northeastern Brazil. J. Infect. Dis., 180:167-175.

11. Peng, M.M., Matos, O., Gatei, W., Das, P., Stantic-Pavlinic, M., Bern, C., Sulaiman, I.M., Glaberman, S., Lal, A.A. \& Xiao, L. 2001. A comparison of Cryptosporidium subgenotypes from several geographic regions. J. Eukaryot. Microbiol., 48:28S-31S.

12. Strong, W.B., Gut, J. \& Nelson, R.G. 2000. Cloning and sequence analysis of a highly polymorphic Cryptosporidium parvum gene encoding a 60 kilodalton glycoprotein and characterization of its 15- and 45-kilodalton zoite surface antigen products. Infect. Immun., 68:4117-34.

13. Sulaiman, I.M., Lal, A.A. \& Xiao, L. 2001. A population genetic study of the Cryptosporidium parvum human genotype parasites. J. Eukaryot. Microbiol., 48:24S-27S.

14. Xiao, L., Bern, C., Limor, J., Sulaiman, I., Roberts, J.. Checkley, W., Cabrera, L., Gilman, R.H. \& Lal, A.A. 2001. Identification of 5 types of Cryptosporidium parasites in children in Lima, Peru. J. Infect. Dis., 183:492-497.

15. Xiao, L., Bern, C., Sulaiman, I.M. \& Lal, A.A. 2004. Molecular epidemiology of human cryptosporidiosis. In: Thompson, R.C.A. (ed.), Cryptosporidium: From Molecules to Disease, Elsevier, p. 121-146. 